\title{
Analytical Study of Neighborhood Discovery and Link Management in OLSR
}

\author{
Evgeny Khorov, Anton Kiryanov, Andrey Lyakhov, Dmitry Ostrovsky \\ Intstitute for Information Transmission Problems \\ Russian Academy of Sciences \\ Moscow, Russia \\ Email: \{khorov, kiryanov, lyakhov, ostrovsky\}@iitp.ru
}

\begin{abstract}
In mobile ad-hoc networks (MANETs), neighborhood discovery and link management (NDLM) mechanism is used by each node to determine the set of its neighbors, or in other words, to establish one-hop links. Values of this mechanism parameters dramatically affect network performance, especially when real-time traffic is transmitted with proactive routing protocols, which imposes constraints on link quality, i.e. reliability, stability and decision-making latency. The efficiency of NDLM mechanisms is usually evaluated by simulation.

The core contribution of this paper is an analytical study of NDLM mechanism defined in OLSR protocol specification. With the developed analytical model of the mechanism, we find the set of parameter values which allow to meet the constraints on link quality indices. By simulation, we show that the usage of these values improves the performance of the network loaded with voice traffic.
\end{abstract}

\section{INTRODUCTION}

Both the growing number of conferences on Mobile Ad-hoc NETworks (MANET) and the number of deployed MANETs prove high demand on this technology which can be applied in a vast set of scenarios. In both static and mobile scenarios, the quality of the wireless channel is constantly changing, which makes neighborhood discovery and link management (NDLM) crucial issues in MANETs. To achieve high throughput and to meet QoS requirements (e.g. while transmitting real-time data), only stable and reliable (providing high probability of successful packets transmission) links shall be used by routing protocols. While reactive routing protocols, e.g. AODV [1], test the channel by transmitting route request packets on demand, proactive protocols, such as OLSR [2], estimate link quality periodically by broadcasting (without relaying) special packets called HELLO (also referred to as beacons). When these packets are received by a node, an originator node may be considered as a neighbor and a link may be established. In mobile scenarios, NDLM is complicated by the following issue: neighbor nodes shall discover each other and open a link between them just after they enter the transmission area of each other and much before they leave it.

NDLM developers face two challenges connected with: 1) the rules of sending HELLOs and 2) the decision making algorithms which determine whether the link shall or shall not be used by the routing protocol.

The first challenge is widely discussed in the literature. In [3], Huang et al. study the influence of HELLO_INTERVAL, i.e. the interval between two consecutive HELLOs, on the network throughput. Short HELLO_INTERVAL results in quick neighborhood discovery but increases the overhead and degrades network throughput.

To improve network performance in mobile scenarios, Voorhaen and Blondia [4] suggest to use short HELLOs which are sent more frequently than default HELLOs. Moreover, nodes change states of links with their neighbours on receiving/missing only one HELLO. This strategy hastens the protocol reaction on topology changes but also causes fluctuation of link states which increases the probability of routing error.

In [5], Nayebi et al. study link management efficiency indices such as link lifetime, discovery delay (the interval between the moment when a node enters the transmission area of another one and the moment when it is discovered), and dead time (the delay of detecting a broken link). The authors compare different beacons schemes: when the interval between consecutive beacons is fixed and when it is exponentially distributed. In the paper, fixed intervals are shown to be more efficient according to the considered indices. A major drawback of that analysis is the raw error rate model: the probability of packet transmission failure is considered to be either 0 or 1 depending on the distance between nodes, while in real networks, it increases continuously from 0 to 1 with the distance between nodes.

The second challenge is less discussed but different decision making algorithms can be found in the specification of well known OLSR protocol ${ }^{1}$.

In OLSR, HELLOs are sent periodically by every node and contain information about all of its open links. Open links may be unidirectional or symmetric. Two nodes open unidirectional links between each other independently using one of the decision making algorithms described in the next paragraph. Symmetric links are opened as follows. Node A considers the link $\mathbf{A} \rightarrow \mathbf{B}$ symmetric if (a) this link is opened by $\mathbf{A}$ and (b) the link $\mathbf{B} \rightarrow \mathbf{A}$ has also been opened according to the last HELLO sent by $\mathbf{B}$ and received by $\mathbf{A}$. Only symmetric links are used by the routing protocol.

${ }^{1}$ In the first version, neighborhood discovery and link management mechanism was described in OLSR specification. Afterwards it was published in a standalone specification called NHDP [6], which is referred by OLSRv2 [7]. 
According to the simplest decision making algorithm described in the specification [2], $\mathbf{A}$ opens unidirectional link $\mathbf{A} \rightarrow \mathbf{B}$ on receiving a HELLO sent by $\mathbf{B}$. A closes the link when the NEIGHB_HOLD_TIME timeout since the last B's HELLO receipt expires. In other words, A closes the link when several B's HELLOs in a row are lost.

A more complicated algorithm uses the link quality value which increases when a HELLO is received and decreases once per HELLO_INTERVAL if no HELLO is received. When this value reaches the upper threshold, the node opens the link. When it falls down to the lower one, the node closes the link. Two thresholds provide the so called link quality hysteresis, which improves links stability.

Another algorithm used in TBRPF [8] also provides link quality hysteresis by opening a link with a neighbor if HELLO_ACQUIRE_COUNT of the last HELLO_ACQUIRE_WINDOW HELLOs sent by the neighbor node are received. If HELLO_ACQUIRE_COUNT = HELLO_ACQUIRE_WINDOW, we obtain an approach proposed in [9], [10] and studied in this paper.

The lack of papers studying the efficiency of decision making algorithms does not allow to compare them, choose the best one and configure it optimally. Being a part of a routing protocol, NDLM mechanisms are often studied by simulation or testbed experiments [11], which are complex and expensive. Moreover, due to the usage of high-level performance indices (e.g. throughput, packet delivery ratio), which are influenced not only by NDLM parameters but also by many other factors, these experiments provide results relevant only to particular scenarios, protocols etc.

To overcome this problem, several papers introduce NDLM efficiency indices [5], [9], [10] and constraints on their values. In [10], we propose an analytical method to configure Mesh Peering Management Protocol (MPMP), a NDLM mechanism specified in IEEE 802.11s mesh networks standard. With the proposed mathematical model, we study the efficiency of different strategies used in MPMP implementations and configure MPMP for different scenarios. By simulation, we show that MPMP parameters values obtained for each scenario with analytical model significantly improve network performance loaded with real-time flows.

Being an extension of [10], this paper studies OLSR NDLM algorithm which opens link just after $r$ HELLOs in a row have been received and closes it after $m$ HELLOs in a row have been missed. The simplest decision making algorithm described in OLSR specification is the special case of this algorithm. Section II gives a simplified description of OLSR NDLM state machine. MPMP differs from OLSR NDLM by a handshake procedure which synchronises the link state at both neighbor nodes. Due to this procedure, the total amount of time when links are unidirectional is negligibly small. OLSR does not have such a mechanism, so the time intervals when the link is unidirectional cannot be ignored. This peculiarity does not allow us to use MPMP analytical model for studying OLSR NDLM mechanism efficiency even with the similar decision making mechanism. So in Section III, we give a brief

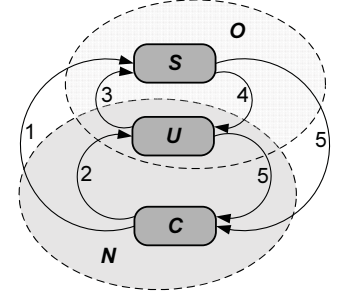

Fig. 1. OLSR NDLM state machine

description of efficiency indices and constraints on them, and in Section IV, we develop a novel analytical model of OLSR NDLM which allows to find values of the indices. Section V shows how to configure OLSR NDLM using the developed model and proves that the selected parameters values reduce the probability that QoS requirements for real-time traffic are not met. Final remarks are given in Section VI.

\section{OLSR NDLM STATE MACHINE}

In this Section, we describe the simplified state machine of OLSR NDLM mechanism studied in the paper.

Let us consider two nodes, $\mathbf{A}$ and $\mathbf{B}$, and a link between them.

As mentioned in Section I, link may be in the following states: $U$ (unidirectional), $S$ (symmetric) and $C$ (closed). Let us state $O$ (opened) be the union of $U$ and $S$, and state $N$ be the union of $U$ and $C$. When the state is $O$, the node announces the link and the link state in its HELLOs.

As OLSR does not synchronize link states, the states of the link at nodes $\mathbf{A}$ and $\mathbf{B}$ may differ. For short, we further refer to the state of the link at node $\mathbf{A}$ and at node $\mathbf{B}$ as $\mathbf{A}$ 's state and B's state, respectively.

Let us consider a node, say $\mathbf{A}$, and focus on the rules of its state transitions (see Fig. 1):

1) If $\mathbf{A}$ 's state is $C$, it receives $r$ HELLOs in a row, and, according to the last of them, B's state is $O$, then $\mathbf{A}$ transits to $S$.

2) If A's state is $C$, it receives $r$ HELLOs in a row, and, according to the last of them, B's state is $C$, then $\mathbf{A}$ transits to $U$.

3) If A's state is $U$ and it receives one HELLO, according to which B's state is $O$, then A transits to $S$.

4) If A's state is $S$ and it receives one HELLO, according to which B's state is $C$, then $\mathbf{A}$ transits to $U$.

5) If A's state is $O$ and it misses $m$ HELLOs in a row, then it transits to $C$.

\section{EFFICIENCY INDICES}

In this section, we give a brief overview of efficiency indices proposed in [10] and constraints on them.

As mentioned above, an effective NDLM mechanism shall open only reliable and stable links with small decision-making latency. 
a) Link reliability: the probability $p$ of successful packet transmission through a link used by the routing protocol must be higher than some predefined threshold $p_{0}$. As only symmetric links are used, let us consider probability $P_{S}(p)$ that the node state is $S$ with given $p$, i.e. the node considers the link as symmetric. [10] gives the following constraint:

$$
P_{S}\left(p_{o}\right) \approx 1 / 2 \text {. }
$$

Given $p$, let $T_{S}(p)$ and $T_{N}(p)$ be the average durations of the time interval, when the state is $S$ and $N$, correspondingly. The transition between $S$ and $N$ is an On-Off process, which yields (see, e.g. [12]):

$$
P_{S}(p)=\frac{T_{S}(p)}{T_{S}(p)+T_{N}(p)} .
$$

b) Link stability: if $p$ remains constant, link state must not fluctuate, i.e. transitions between $S$ and $N$ must rarely occur. Link fluctuation is defined as

$$
g(p)=\frac{1}{T_{S}(p)+T_{N}(p)} .
$$

The value $\frac{1}{2 g(p)}$ is the average duration of time interval between two consecutive changes of the state. For routing information to be correct and up-to-date, it is necessary that

$$
\forall p<1 \Rightarrow \frac{1}{2 g(p)} \gg T_{\text {update }},
$$

where $T_{\text {update }}$ is the topology update interval.

c) Decision-making latency: the link should be established after $p$ exceeds the predefined threshold $p_{0}$ and much earlier than it becomes unreliable again. In other words, the following requirement shall be met:

$$
T_{\text {delay }} \ll T_{\text {link }},
$$

where $T_{\text {delay }}$ is the interval between the moment when $p$ reached the threshold $p_{0}$ and the moment when the link was established, and $T_{l i n k}$ is the physical link lifetime, i.e. the average time period while $p \geq p_{0}$. In networks with moderate mobility where $p$ changes gradually with time, $T_{\text {delay }} \sim T_{N}\left(p_{0}\right)$, where $T_{N}(p)$ is the mean duration of state $N$.

In the next section, we develop the analytical model of OLSR NDLM mechanism which allows us to find $P_{S}(p), g(p), T_{N}(p)$ and thus to check if all constraints introduced in this section are satisfied.

\section{Mathematical Model}

We develop analytical model as follows. In the beginning, we evaluate the probability $P_{S}(p)$ that the state is $S$. After that, we estimate the mean duration $T_{S}(p)$ of state $S$. Taking (2) and (3) into account, one can easily obtain $g(p)$ and $T_{N}(\mathrm{p})$ as follows:

$$
g(p)=\frac{P_{S}(p)}{T_{S}(p)}, \quad T_{N}(p)=\frac{1}{g(p)}-T_{S}(p) .
$$

\section{A. Estimation of the Probability of State $S$}

To obtain $P_{S}(p)$, we evaluate the probability $P_{O}(p)$ that a node, say $\mathbf{A}$, is in state $O$.

A's transitions between states $O$ and $C$ are independent from the state of $\mathbf{B}$ because they depend only on the fact whether B's HELLOs were received or lost but not on their content. When $r$ HELLOs in a row are received, $\mathbf{A}$ transits from $C$ to $O$. When $m$ consecutive HELLOs are lost, it transits from $O$ to $C$.

This transition process denoted as $J_{O C}(t)$ is a discretetime On-Off [12] process with time unit HELLO_INTERVAL. Given the average durations $T_{O}(p)$ and $T_{C}(p)$ of states $O$ and $C$, respectively, $P_{O}(p)$ may be found as follows:

$$
P_{O}(p)=\frac{T_{O}(p)}{T_{O}(p)+T_{C}(p)} .
$$

Let us find $T_{O}(p)$. Suppose that $\mathbf{A}$ receives a HELLO from $\mathbf{B}$ and transits to state $O$ at moment $t_{0}=0$. Consider Markov process $J_{O}(t)$ with states $C, O^{(j)}, \quad j \in\{0,1, . ., m-1\}$, where $j$ indicates how many HELLOs $\mathbf{A}$ has missed in a row. State $O^{(0)}$ is initial. State $C$ is absorbing. Obviously, $T_{O}$ is the mean duration of process $J_{O}(t)$.

Consider a subprocess of $J_{O}(t)$ starting with state $O^{(0)}$ and finishing with the first transition to either $O^{(0)}$ or $C$, which we refer to as cycle. The cycle is successful if its final state is $O^{(0)}$; otherwise, it is unsuccessful. According to these definitions, the reception of a HELLO means the end of a cycle and the beginning of another one. The cycle is unsuccessful if $m$ HELLOs in a row are lost. So the duration of an unsuccessful cycle equals $m$ and the probability that a cycle is unsuccessful equals $\pi=(1-p)^{m}$.

The probability that a cycle is successful equals $1-\pi$. Duration $k$ of a successful cycle may equal $1,2, . ., m$ with conditional probability $p_{k}=\frac{(1-p)^{k-1} p}{1-\pi}$.

To evaluate $T_{O}(p)$, we apply the technique of generating functions. At first, we set down the generating functions for durations of successful and unsuccessful cycles and also the generating function for the number of successful cycles in $J_{O}(t)$. After that, we construct the generating function for the total duration of $J_{O}(t)$ and, finally, evaluate $T_{O}$.

The generating function of the successful cycle duration equals:

$$
f(z)=\sum_{k=1}^{m} p_{k} z^{k}=\frac{p z\left(1-z^{m} \pi\right)}{(1-\pi)(1-z(1-p))} .
$$

The generating function of the unsuccessful cycle duration is $g(z)=z^{m}$.

Process $J_{O}(t)$ consists of several successful cycles and the final unsuccessful one. The probability that the number of successful cycles is $n$ equals $\pi(1-\pi)^{n}$. In other words, the number of successful cycles is distributed geometrically with parameter $\pi$, which means that its generating function is:

$$
F(z)=\frac{\pi}{1-(1-\pi) z}=\frac{(1-p)^{m}}{1-\left(1-(1-p)^{m}\right) z} .
$$


The generating function of the total duration of all successful cycles is $F(f(z))$ (see, e.g. [13]). Hence, the generating function of the overall duration of $J_{O}(t)$ is as follows:

$$
\Omega_{0}(z)=F(f(z)) \cdot g(z)=\frac{(1-p)^{m}(1-z(1-p)) z^{m}}{1-z+z^{m+1} p(1-p)^{m}} .
$$

By differentiating (8) and substituting $z=1$, we obtain Lemma 1.

Lemma 1 The mean duration $T_{O}(p)$ of state $O$ is defined by:

$$
T_{O}(p)=\frac{1-(1-p)^{m}}{p(1-p)^{m}}
$$

Substitutions $O \rightarrow C, p \rightarrow 1-p$ and $m \rightarrow r$ provide Lemma 2.

Lemma 2 The mean duration $T_{C}(p)$ of state $C$ is defined by:

$$
T_{C}(p)=\frac{1-p^{r}}{(1-p) p^{r}}
$$

Given $T_{O}(p)$ and $T_{C}(p), P_{O}(p)$ is defined by (7).

Finally, using Lemma 3, we obtain $P_{S}(p)$ :

Lemma $3 P_{S}(p)$ is defined as follows:

$$
P_{S}(p)=P_{O}^{2}(p)
$$

Proof: The state of node $\mathbf{A}$ is $S$ if and only if all of the following events occur:

1) $\mathbf{A}$ is in state $O$;

2) $\mathbf{B}$ was in state $O$ at the moment when $\mathbf{A}$ received a HELLO from $\mathbf{B}$ for the last time.

The probability of each event is $P_{O}(p)$. The claim of Lemma 3 is justified since events 1) and 2) are independent.

\section{B. Evaluation of the Average Duration of $S$}

Consider two independent processes of nodes $\mathbf{A}$ and $\mathbf{B}$ transitions between states $O$ and $C$ defined in Section IV-A, which we refer to as $J_{O C}^{(A)}(t)$ and $J_{O C}^{(B)}(t)$. Let us define process $J_{S^{*} N^{*}}(t)$, which is in state $S^{*}$ if both $J_{O C}^{(A)}(t)$ and $J_{O C}^{(B)}(t)$ are in state $O$. Otherwise, $J_{S^{*} N^{*}}(t)$ is in state $N^{*}$.

$J_{S^{*} N^{*}}(t)$ approximates the processes of A's and B's transitions between $S$ and $N$. Two following factors result in deviation. Firstly, A obtains information about B's transition in state $O$ only with a HELLO sent by B. In other words, A transits to $S$ with some delay, which grows inversely to $p$. Secondly, A's transition to $N$ caused by B's transition to $C$ holds with a similar delay. Anyway, as shown in Section $\mathrm{V}$, these delays compensate each other to a considerable degree, so we can quite accurately evaluate $T_{S}(p)$ by the mean duration $T_{S^{*}}(p)$ of state $S^{*}$.

The duration of state $O$ has the same distribution for both processes $J_{O C}^{(A)}(t)$ and $J_{O C}^{(B)}(t)$ and the same mean referred to as $T_{O}(p)$.
Lemma 4 Given $T_{O}(p), T_{S^{*}}(p)$ is defined by

$$
T_{S^{*}}(p)=\frac{T_{O}(p)}{2} .
$$

Proof: Let $f_{t}$ be the discrete distribution of state $O$ duration, and $F(t)$ be its c.d.f. Suppose that $J_{S^{*} N^{*}}(t)$ changes its state to $S^{*}$ at moment $t_{0}$. Without the loss of generality, we assume that $J_{O C}^{(A)}(t)$ transits to $O$ at $t_{0}$ and $J_{O C}^{(B)}(t)$ has been in $O$ by that moment.

Let us consider the time interval during which process $J_{O C}^{(B)}(t)$ is staying in state $O$ and which includes moment $t_{0}$. Obviously, the probability that the duration of this interval is $\tau$ equals $\frac{\tau f_{\tau}}{T_{O}(p)}$. Since processes $J_{O C}^{(A)}(t)$ and $J_{O C}^{(B)}(t)$ are statistically independent, $t_{0}$ equiprobably corresponds to any moment of the interval. Consider the part of that interval starting from moment $t_{0}$. We obtain that the p.d.f. of the part duration is defined by the following equation:

$$
g(t)=\sum_{\tau>t} \frac{\tau f_{\tau}}{T_{O}(p)} \cdot \frac{1}{\tau}=\frac{(1-F(t))}{T_{O}(p)} .
$$

P.d.f. $g(t)$ corresponds to the following c.d.f.:

$$
G(t)=\frac{1}{T_{O}(p)} \int_{0}^{t}(1-F(x)) d x .
$$

$J_{S^{*} N^{*}}(t)$ transits from $S^{*}$ with the first exit from $O$ of either $J_{O C}^{(A)}(t)$ or $J_{O C}^{(B)}(t)$. Consequently, the probability that the duration of state $S^{*}$ is not less than $x$ is $(1-F(x))(1-G(x))$. By the properties of nonnegative random variables [13], we obtain:

$$
T_{S^{*}}(p)=\int_{0}^{\infty}(1-F(x))(1-G(x)) d x .
$$

Since $\frac{d}{d x}(1-G(x))=-\frac{1-F(x)}{T_{O}(p)}$, it holds:

$$
T_{S^{*}}(p)=-\left.\frac{T_{O}(p)}{2}(1-G(x))^{2}\right|_{0} ^{\infty}=\frac{T_{O}(p)}{2} .
$$

From Lemma 4, we obtain:

$$
T_{S^{*}}(p)=\frac{T_{O}(p)}{2}=\frac{1-(1-p)^{m}}{2 p(1-p)^{m}} .
$$

By applying (6)-(7) and (9)-(12), we evaluate the values of OLSR NDLM efficiency indices.

\section{NUMERICAL RESULTS}

\section{A. Model Validation}

To validate the model developed in Section IV, we compare its results with the results obtained with well-known network simulator ns-3 [14]. We consider two nodes both configured with default values of OLSR and Wi-Fi (IEEE 802.11a) parameters. We use TwoRayGroundPropagationLossModel along with NakagamiPropagationLossModel $\left(m_{0}=1.5, m_{1}=\right.$ $m_{2}=0.75$ ) both implemented in ns- 3 . By varying the distance 


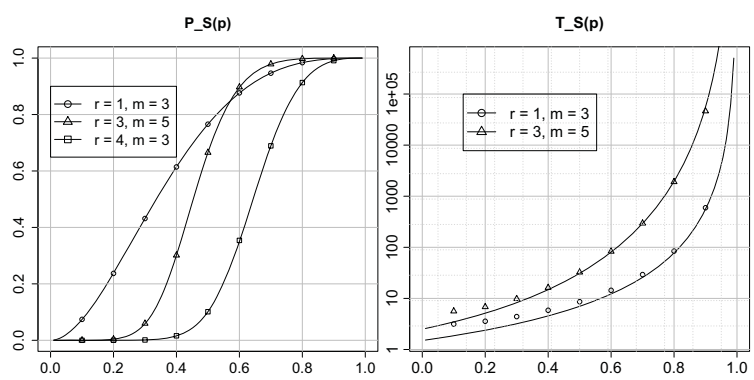

Fig. 2. Comparison of the results obtained with analytical modelling (curves) and simulation (points) for $P_{S}(p)$ and $T_{S}(p)$

between them, we change the probability of successful packet transmission. For each value of $p$, we observe the link between nodes during quite a long time interval $(10000 \mathrm{~s}$, while HELLO_INTERVAL is $2 \mathrm{~s}$ ). To obtain statistically significant results for the values of $T_{S}(p)$ and $P_{S}(p)$, we run simulation for 50 times at each point. Then we change the values of OLSR NDLM parameters and repeat the experiment. The analytical and simulation results match with good precision in a wide range of $r, m, p$ values except for the cases when $p$ is small, see Fig. 2. However, with small $p$, the link is closed almost always, so, in practice, these cases are not of interest.

\section{B. OLSR NDLM Mechanism Adjusting}

Let us demonstrate how to configure OLSR for MANET loaded with real-time voice traffic.

We use G.729 [17] audio codec which generates 50 packets per second. According to the ITU recommendation [18], the quality of the received voice signal depends on the average packet delivery time, the jitter, i.e. the variation of the delivery time, and the packet loss ratio $(P L R)$. In nonoverloaded IEEE 802.11 networks, the actual packet delivery time is much less than the required one, and $P L R$ becomes the most important factor [19]. According to [20], the quality of G.729 voice signal is not fair if end-to-end $P L R$ is higher than $10 \ldots 12 \%$. As $P L R$ changes with time, we measure it every interval $\Delta=$ $1 \mathrm{sec}$ and define the unavailability of voice service, $N V$, as the probability that end-to-end $P L R_{\Delta}$ during $\Delta$ is higher than $10 \%$, i.e. more than 5 packets of a flow are lost. As $k$ packets are lost with probability $\frac{50 !}{(50-k) ! k !} P L R^{k}(1-P L R)^{50-k}$, to obtain low $N V$, say $N V=1 \%$, one shall provide $P L R$ lower than $3 \%$.

End-to-end $P L R$ is calculated as follows: $P L R=1-$ $\left(1-\operatorname{per}^{\rho+1}\right)^{D}$, where per is packet error rate, $\rho=7$ is IEEE 802.11 retry threshold, and $D$ is the number of hops between the originator and the receiver of a voice flow. E.g. if $D \leq 5$ for any route in the network, to achieve $P L R \leq 3 \%$ NDLM mechanism shall open links with $1-$ per $\gtrsim p_{0}=0.5$.

Let $L$ be such a distance between 2 STAs that the probability of successful packet transmission between them is $p(L)=0.5$. We locate 50 mobile nodes in the area $2.3 L \times 2.3 L$. With such high node density, the network is almost always connected and
TABLE I

CHOOSING THE VALUES OF OLSR NDLM PARAMETERS

\begin{tabular}{|c|c|c|c|c|}
\hline Velocity & $T_{\text {link }}$ & Chosen $(r, m)$ & $\frac{T_{N}\left(p_{0}\right)}{T_{\text {link }}}$ & $\frac{T_{\text {update }}}{\frac{1}{2 g_{\max }}}$ \\
\hline $10^{-4}$ & 12325 & $(5,6)$ & 0.014 & 0.014 \\
$5 \cdot 10^{-4}$ & 2465 & $(4,5)$ & 0.030 & 0.034 \\
$2 \cdot 10^{-3}$ & 616 & $(3,4)$ & 0.062 & 0.065 \\
$10^{-2}$ & 123 & $(2,3)$ & 0.14 & 0.06 \\
\hline
\end{tabular}

almost all routes are not longer than $D=5$ hops. The nodes move according to 2D Random Direction Mobility Model [21] with velocity $v=\left\{10^{-4}, 5 \cdot 10^{-4}, 2 \cdot 10^{-3}, 10^{-2}\right\} L$ per HELLO_INTERVAL, so the time interval when the distance between 2 nodes is less than $L$ is $T_{\text {link }}=\frac{\pi^{2} L}{8 v}$ [5].

The nodes work under OLSR protocol which obtains topology information about 2-hop neighborhood from HELLOs and about the other part of the network from TC packets. As OLSR is a hop-by-hop routing protocol, we consider $T_{\text {update }}=$ HELLO_INTERVAL, which guaranties that the information about links at least in 2-hop neighborhood is stable and provides low probability of routing errors. We could choose $T_{\text {update }}$ to be equal to the interval between TC packets, which is 2.5 times higher than HELLO_INTERVAL. But as it is shown below, there is no need to determine $T_{\text {update }}$ exactly.

Thus, having determined $p_{0}, T_{\text {link }}$, and $T_{\text {update }}$, we can adjust OLSR NDLM mechanism.

At the first step, using (9)-(11), we find pairs $(r, m)$ which meet (1). They are $(2,3),(3,4),(4,5),(5,6), \ldots$

At the second step, we find $T_{N}\left(p_{0}\right)$ and $g_{\max }=\max g(p)$ for each pair chosen above. As final values of OLSR NDLM parameters we may choose any pair which satisfies (4) and (5). For example (see Table I), we choose such a pair that

$$
\frac{T_{N}\left(p_{0}\right)}{T_{\text {link }}} \sim \frac{T_{\text {update }}}{\frac{1}{2 g_{\max }}}
$$

We run simulation using ns-3 [14] environment and prove that chosen pairs $(r, m)$ provide better results than other values of OLSR parameters. Fig. 3 (a) shows that the usage of the parameters values from Table I ("opt") provides that voice service is available almost always while the usage of default OLSR configuration $(1,3)$ leads to the unavailability of voice service higher than $20 \%$ ("def").

Fig. 3 (b)-(d) show that the method proposed in the paper allows to find the values of OLSR NDLM parameters which decrease the unavailability of voice service, i.e. improve network performance.

The second result obtained with simulation is that several pairs provide almost the best network efficiency. E.g., when the velocity equals $10^{-4}$ the usage of pairs $(5,4),(5,5),(5,6),(6,6)$, $(6,7)$, etc. results in almost the same performance. It means that there is no need to determine $p_{0}, T_{\text {update }}$ and $T_{\text {link }}$ exactly and to meet (1) and (13) strictly. Any parameters values which meet restrictions considered in Section III provide good results.

Despite the optimal parameters values depend at least on the velocity, the usage of $r=3, m=4$ leads to high network 


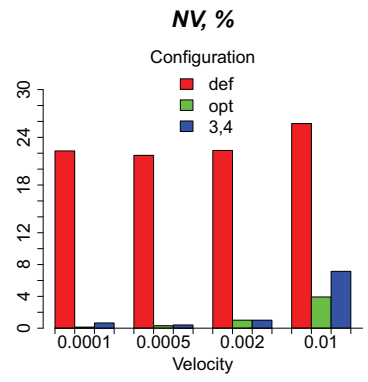

(a)

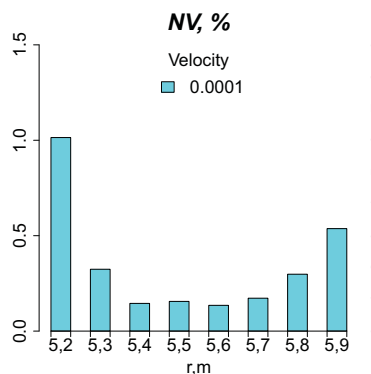

(c)

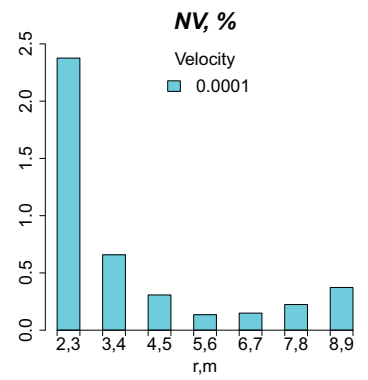

(b)

NV, \%

Velocity

$\square 0.0001$

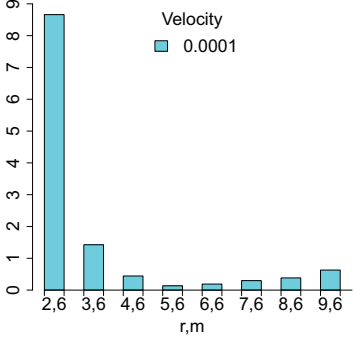

(d)
Fig. 3. The unavailability of voice service in different scenarios

performance in all considered scenarios, see Fig. 3a. This fact, even leading to suboptimal results, is quite valuable for the real system design.

\section{CONCLUSION}

In this paper, we have considered theoretical aspects of neighborhood discovery and link management and developed an analytical model of decision-making algorithm used in OLSR NDLM mechanism. Based on efficiency criteria proposed in [10] we have applied the model to configure the mechanism to achieve the best network performance. Apart from being useful to adjust OLSR NDLM optimally without long expensive testbed experiments or simulation, the proposed approach is quite valuable to answer the question which NDLM mechanism is better: described in OLSR or IEEE 802.11s specification, which motivates us (a) to compare OLSR and IEEE 802.11s NDLM mechanisms (b) to compare different NDLM decision making algorithms. This work will be done in the nearest feature.

If OLSR NDLM mechanism outperforms NDLM mechanism of IEEE $802.11 \mathrm{~s}$ mesh networks, one shall replace NDLM mechanism in mesh devices, which may be done using FLAVIA [22] architecture to ensure compatibility with legacy devices.

\section{ACKNOWLEDGMENT}

This work is partially supported by EU FP7 FLAVIA project [22] and by research project "QoS provisioning methods for the access to broadband multimedia services in wireless selforganized networks" (\#2012-1.2.1-12-000-2006-009) of the Ministry of Education and Science of Russian Federation.

\section{REFERENCES}

[1] C. Perkins, E. Belding-Royer, S. Das. Ad hoc On-Demand Distance Vector (AODV) Routing. IETF, July 2003. http://tools.ietf.org/html/rfc3561

[2] P. Jacquet, T. Clausen, Optimized Link State Routing Protocol (OLSR). IETF, October 2003. http://www.ietf.org/rfc/rfc3626.txt

[3] Y. C. Huang, S. Bhatti, D. Parker. Tuning OLSR. Proceedings of PIMRC2006 - The 17th Annual IEEE International Symposium on Personal, Indoor and Mobile Radio Communications. Helsinki, Finland, 2006.

[4] M. Voorhaen, C. Blondia. Analyzing the impact of neighbor sensing on the performance of the OLSR protocol. Proceedings of 4th Intl. Symposium on Modeling and Optimization in Mobile, Ad Hoc, and Wireless Networks (WiOpt06). Boston, Massachusetts, 2006.

[5] A. Nayebi, G. Karlsson, H. Sarbazi-Azad. Evaluation and design of beaconing in mobile wireless networks. Ad Hoc Networks, 2011, vol. 9, no. 3, pp. 368-386.

[6] T. Clausen, C. Dearlove, J. Dean. Mobile Ad Hoc Network (MANET) Neighborhood Discovery Protocol (NHDP). IETF, April 2011. http://tools.ietf.org/html/rfc6130

[7] T. Clausen, C. Dearlove, P. Jacquet, U. Herberg. The Optimized Link State Routing Protocol version 2 Internet-Draft. IETF, May 2012. http://tools.ietf.org/html/draft-ietf-manet-olsrv2-15

[8] R. Ogier, F. Templin, M. Lewis. Topology Dissemination Based on Reverse-Path Forwarding (TBRPF). IETF, February 2004. $\mathrm{http}: / /$ tools.ietf.org/html/rfc 3684

[9] A. Kiryanov, A. Lyakhov, A. Safonov, E. Khorov. A method to estimate efficiency of the connection control mechanisms in wireless self-organizing networks. Automation and Remote Control, 2012, vol. 79, no. 2, pp. 797-809.

[10] E. Khorov, A. Kiryanov, A. Lyakhov, A. Safonov. Analytical study of link management in IEEE 802.11s mesh networks. Proceedings of International Symposium on Wireless Communication Systems (ISWCS). France, August 28-31, 2012.

[11] H. M. Ali, A. M. Naimi, A. Busson, V. Vèque. An efficient link management algorithm for high mobility mesh networks. Proceedings of the 5th ACM international workshop on Mobility management and wireless access. Greece, 2007.

[12] P. Jelencovic, P. Momcilovic. Asymptotic loss probability in a finite buffer fluid queue with heterogeneous heavy-tailed On-Off processes. Annals of Applied Probability, 2003, vol. 13, no. 2, pp. 576-603.

[13] W. Feller. Introduction to probability theory and its applications, vol. 2, 3rd edition. Wiley: 1968.

[14] The ns-3 network simulator. http://www.nsnam.org/

[15] IEEE Std 802.11s, IEEE Standard for local and metropolitan area networks, part 11: Wireless LAN Medium Access Control (MAC) and Physical Layer (PHY) Specifications. Amendment: Mesh Networking.

[16] IEEE Std 802.11-2007 (Revision of IEEE Std 802.11-1999), IEEE Standard for Local and metropolitan area networks, part 11: Wireless LAN Medium Access Control (MAC) and Physical Layer (PHY) Specifications.

[17] Recommendation G.729 - Coding of speech at $8 \mathrm{kbit} / \mathrm{s}$ using conjugatestructure algebraic-code-excited linear prediction (CS-ACELP). ITUT, January 2007.

[18] Recommendation G.107 - The E-Model - A Computational Model In Use In Transmission Planning. ITU-T, March 2005.

[19] E. Khorov, A. Safonov. Multiple metrics in MANET with end-to-end QoS support for unicast and multicast traffic. Proceedings of the Third international conference on Multiple access communications (MACOM2010). Berlin, 2010.

[20] K. Perlicki. Simple analysis of the impact of packet loss and delay on voice transmission quality. Journal of Telecommunications And Information Technology, February 2002.

[21] M Izuan, M Saad, Z. A. Zukarnain. Performance Analysis of RandomBased Mobility Models in MANET Routing Protocol. European Journal of Scientific Research, 2009, vol. 32, no. 4, pp. 444-454.

[22] FLexible Architecture for Virtualizable future wireless Internet Access (FLAVIA). http://www.ict-flavia.eu/ 